University of New Hampshire

University of New Hampshire Scholars' Repository

Physics Scholarship

Physics

6-15-1997

\title{
A new, temporarily confined population in the polar cap during the August 27, 1996 geomagnetic field distortion period
}

Jiasheng Chen

Theodore A. Fritz

Robert B. Sheldon

Harlan E. Spence

Boston University, harlan.spence@unh.edu

Walther N. Spjeldvik

See next page for additional authors

Follow this and additional works at: https://scholars.unh.edu/physics_facpub

Part of the Physics Commons

\section{Recommended Citation}

Chen, Jiasheng; Fritz, Theodore A.; Sheldon, Robert B.; Spence, Harlan E.; Spjeldvik, Walther N.; Fennell, Joseph F.; and Livi, Stefano, "A new, temporarily confined population in the polar cap during the August 27, 1996 geomagnetic field distortion period" (1997). Geophysical Research Letters. 299.

https://scholars.unh.edu/physics_facpub/299

This Article is brought to you for free and open access by the Physics at University of New Hampshire Scholars' Repository. It has been accepted for inclusion in Physics Scholarship by an authorized administrator of University of New Hampshire Scholars' Repository. For more information, please contact Scholarly.Communication@unh.edu. 


\section{Authors}

Jiasheng Chen, Theodore A. Fritz, Robert B. Sheldon, Harlan E. Spence, Walther N. Spjeldvik, Joseph F. Fennell, and Stefano Livi 


\title{
A new, temporarily confined population in the polar cap during the August 27, 1996 geomagnetic field distortion period
}

\author{
Jiasheng Chen, Theodore A. Fritz, Robert B. Sheldon, Harlan E. Spence, and \\ Walther N. Spjeldvik \\ Center for Space Physics, Boston University, Boston, MA
}

Joseph F. Fennell

The Aerospace Corporation, Los Angeles, CA

Stefano Livi

Max-Planck-Institut fuer Aeronomie, 37189 Katlenburg-Lindau, Germany

\begin{abstract}
On August 27, 1996, a two-hour energetic heavy ion event $(\sim 1 \mathrm{MeV})$ was detected at 8:25 $\mathrm{UT}$ at apogee $\left(\sim 9 R_{e}\right.$ and an invariant latitude of $\left.\sim 80^{\circ}\right)$, by the Charge and Mass Magnetospheric Ion Composition Experiment onboard POLAR. The event, with a maximum spin averaged peak flux of $\sim 150$ particles $/\left(\mathrm{cm}^{2}\right.$ sr-s-MeV), showed three local peaks corresponding to three localized regions; the ion pitch angle distributions in the three regions were different from an isotropic distribution and different from each other. No comparable flux was observed by the WIND spacecraft. The appearance of lower energy $\mathrm{He}^{++}$and $\mathrm{O}^{>+2}$ during the event period indicates a solar source for these particles. From region 1 to 2 to 3 , the helium energy spectra softened. A distorted magnetic field with three local minima corresponding to the three He peak fluxes was also observed by POLAR. A possible explanation is that the energetic He ions were energized from lower energy helium by a local acceleration mechanism that preferred smaller rigidity ions in the high altitude polar cusp region.
\end{abstract}

\section{Introduction}

The investigation of geomagnetically trapped heavy ions began at the 1960's [Krimigis and Van Allen, 1967; Fritz and Krimigis, 1969]. Axford [1970] suggested a direct injection from the polar regions with subsequent acceleration; and Cornwall [1972] and Spjeldvik and Fritz $[1981 \mathrm{a}, \mathrm{b}]$ discussed and modeled charge exchange, Coulomb collisions, and radial diffusive transport. Recently, a trapped ${ }^{3} \mathrm{He}$ species in the inner belt was observed by the CRRES satellite [Chen et al., 1994, $1996 \mathrm{a}, \mathrm{b}]$, and an origin of these ions in energetic proton interactions with the residual atmosphere was suggested [Chen et al., 1996a,b; Selesnick and Mewaldt, 1996]. However, all of the aforementioned discussions regarding sources are limited to within the known radiation belts either near the equator or at lower altitude.

Copyright 1997 by the American Geophysical Union.

Paper number 97GL01369.

0094-8534/97/97GL-01369\$05.00
POLAR was launched on February 24, 1996 with a $1.8 \times 9 R_{e}$ (Earth radius) polar orbit, which has an inclination of $86^{\circ}$ and period of 18 hours. The spacecraft, so far, samples high altitude regions in the north and low altitudes in the south and spins with a period of about 6 seconds. The Charge and Mass Magnetospheric Ion Composition Experiment (CAMMICE) onboard POLAR consists of two sensors, the Heavy Ion Telescope (HIT) and the Magnetospheric Ion Composition Sensor (MICS), designed to measure the charge and mass composition within the magnetosphere over the energy range of $1 \mathrm{KeV} / \mathrm{e}$ to $60 \mathrm{MeV} / \mathrm{ion}$, to determine the fluxes of various ion species and their relative abundances and to seek to identify mechanisms by which these ions are energized and transported from their source populations within geospace. The HIT sensor uses a three-element solid-state detector telescope to measure the rate of energy loss and the ion's total incident energy, and this permits an unambiguous determination of the ion mass and incident energy over the energy range from $100 \mathrm{KeV} /$ ion to $60 \mathrm{MeV} /$ ion. There are also some discrete discriminators on each detector to create data response channels that can be accumulated in a manner identical to a number of previous instruments of this type flown as part of the science payload of Explorer 45, ATS-6, Viking, and CRRES [Fritz and Cessna, 1975; and Fritz et al., 1985]. The data are then sampled every $1 / 16$ of a spin; that is, 16 sectors in each spin.

\section{A Special Event: Observation of August 27, 1996}

Figure 1 displays the spin averaged passband integrated flux of helium $(\sim 0.52-1.15 \mathrm{MeV})$ measured by the HIT sensor during August 27, 1996. At 8:25 UT when the POLAR spacecraft was $9 R_{e}$ from the Earth with MLAT $\simeq 67^{\circ}$, and MLT $\simeq 14.7$ hours, the HIT sensor detected a sudden increasing He flux. This energetic helium event lasted more than two hours with a peak spin averaged flux of about 150 particles $/\left(\mathrm{cm}^{2}-\right.$ sr-s-MeV). The day-side and high latitude location, the energy, and the two-hour time period indicate that this is a yery special event and is indeed very surprising be- 


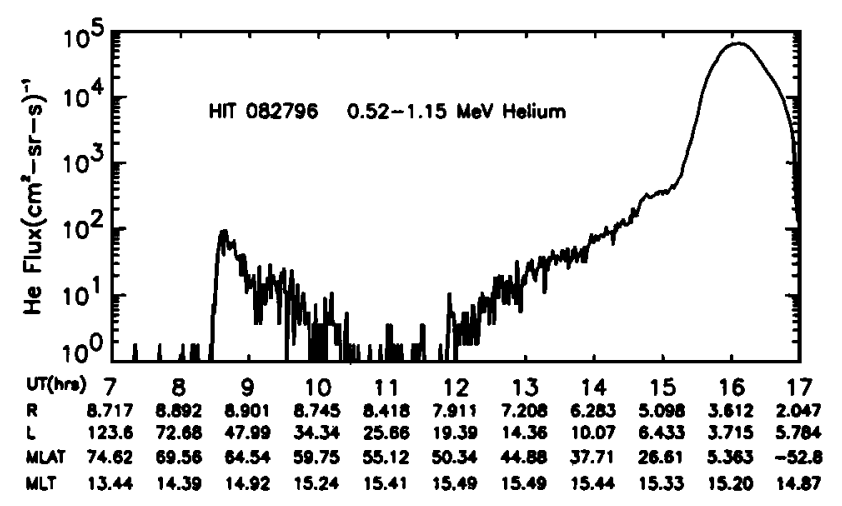

Figure 1. Spin averaged integral flux of helium measured by the CAMMICE/HIT sensor during August 27, 1996. At the bottom, the descriptive ephemeris parameters are the L-shell values, radial distance of the POLAR (R), magnetic latitude (MLAT), and magnetic local time (MLT), respectively.

cause it is unexpected theoretically [e.g., Ilyin et al., 1986; Chen et al., 1996b]. About 3.5 hours after event onset, an increasing helium ion flux was also measured all the way into $\mathrm{L}=5$.

\section{New Temporarily Confined Population Over the Polar Cap}

A closer look at Figure 1 indicates that the particle fluxes in this event had three different local peaks corresponding to three different regions: before 9.1 UT, 9.1-10 UT, and after $10 \mathrm{UT}$. Figure 2 plots the pitch angle distributions of the helium ions in counts per sample number. The three different regions are showed in the three top panels. In Figure 2, each bin is azimuthally 22.5 degrees wide. The HIT sensor has a small field of view ( $\sim 12^{\circ}$ conical perpendicular to the spin axis) so that for particles trapped in the radiation belt it will

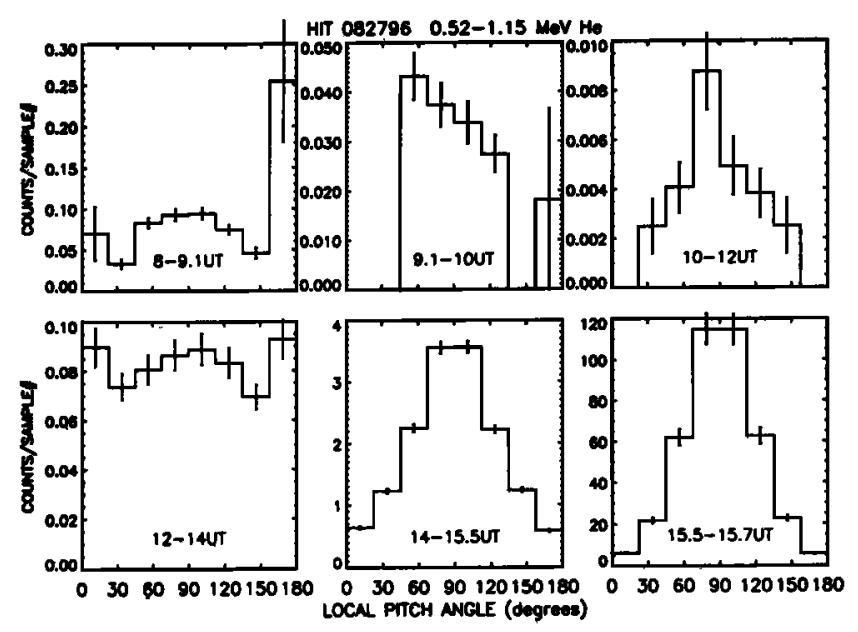

Figure 2. Counts/sample number vs. the computed pitch angles for $\sim 0.52-1.15 \mathrm{MeV}$ helium are displayed as a function of time and correspond to the different $L$ regions showed in Fig. 1. The vertical bars indicate the statistical errors.

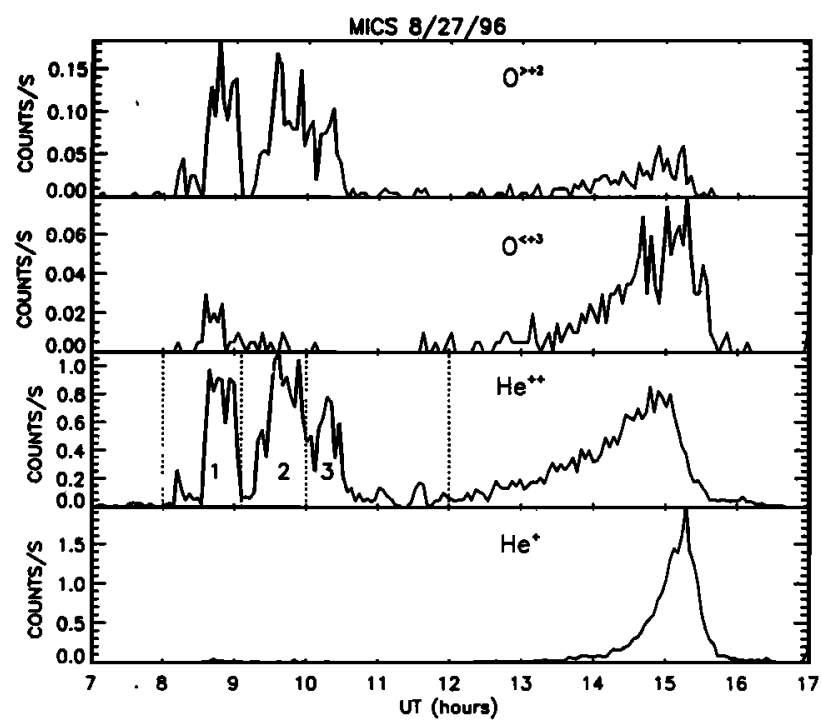

Figure 3. Counting rates measured by the MICS sensor are ordered from top to bottom being $\mathrm{O}^{>+2}, \mathrm{O}^{<+3}$, $\mathrm{He}^{++}$, and $\mathrm{He}^{+}$, respectively. The numbers of 1,2 , and 3 within the vertical dashed lines in $\mathrm{He}^{++}$panel mark the three different regions in this event.

detect a maximum counting rate when its look direction is perpendicular to the magnetic field vector. This is what the bottom-right two panels (14-15.5 UT and 15.5-15.7 UT) show, characteristic of the trapped populations within the radiation belts. In contrast, for an isotropic distribution, a fairly straight horizontal line is expected, independent of HIT look direction as shown in the 12-14 UT panel where, to within the uncertainties, an essentially isotropic distribution of the helium ions was observed. The latter is expected since they are located at $L=10-19$ range beyond the stable trapping region for $\sim 1 \mathrm{MeV}$ helium.

Of particular interest is the top three panels in Figure 2 , which shows that the ion pitch angle distributions of this event in the three regions are different from each other, and all of them were different from an isotropic distribution, indicating a temporarily confined component.

Both Figures 1 and 2 show that the observed energetic helium ions in the polar cap region are spatially separated from those close to the equator in the radiation belt. A natural question is what is the original source for the energetic population in the polar cap region and what maintains the confining topology?

The counting rates of lower energy ions measured by the MICS sensor are shown in Figure 3. The figure exhibits large amounts of lower energy $\mathrm{He}^{++}$and $\mathrm{O}^{>+2}$ during the two-hour event period, thus suggesting a solar wind source for the particle fluxes we report. Another interesting point is that the $\mathrm{He}^{++}$panel also displays a different peak in each of the three regions in this event. Comparing Figure 1 with Figure 3, one finds that the helium energy spectra become softened from region 1 to 2 to 3 , because the $\mathrm{He}^{++}$ions measured by the MICS are in the energy range of about $1-200 \mathrm{KeV} / \mathrm{e}$. The key point is that the observed helium ions in this two-hour event most likely are from the same solar wind source but that they exhibit different energy spectra in 
different regions, which suggests a further local acceleration mechanism.

The measurements strongly suggest that the energetic ions in the polar cap constitute a new temporarily confined heavy ion population that was controlled by some local accelerating and confining mechanism. This high latitude discovery warrants further investigation.

\section{Discussion}

Another way to determine the source of the observed energetic helium ions is using the isotopic ratio. The effective solar source ${ }^{3} \mathrm{He} /{ }^{4} \mathrm{He}$ ratio is expected to be a few parts in $10^{4}$ in the solar corona and in the solar wind [e.g., Axford, 1970; Geiss et al., 1970; Geiss and Reeves, 1972], whereas the same ratio was less than one part in $10^{6}$ for plasmas associated with the ionosphere [Axford, 1970]. At L > 5, the direct event mode of the HIT sensor detected 21 helium ions at energy of about 1.5-4.5 MeV, among them five are ${ }^{3} \mathrm{He}$. Although this constitutes rather low count statistics, if they were from solar wind accelerated by interplanetary shocks, one would expect the HIT to detect only $0.01{ }^{3} \mathrm{He}$ not five, that is more than two orders of magnitude less than what was observed. Therefore, if solar wind is the source then there would possibly be a local acceleration mechanism that preferred lower rigidity ions.

The CRRES spacecraft observed a significant amount of ${ }^{3} \mathrm{He}$ at $\mathrm{L}<4$ [Chen et al. 1994], which can be attributed to the energetic proton interactions with the residual atmosphere [Chen et al., 1996a,b; Selesnick and Mewaldt, 1996]. However, within the classical radiation belts the measured ${ }^{3} \mathrm{He} /{ }^{4} \mathrm{He}$ ratios at $51-86$ $\mathrm{MeV} /$ nucleon increased with the decreasing $\mathrm{L}$, and that is different from the present $\mathrm{HIT}$ result $\left({ }^{3} \mathrm{He} /{ }^{4} \mathrm{He}\right.$ decreased as L decreases). Thus, by spatial location and radial gradient, proton interactions by nuclear reactions in the atmosphere is not likely to be the source for the helium ions reported here.

For small (impulsive) solar energetic particle (SEP) events at an energy of $\sim 1-4 \mathrm{MeV} /$ nucleon, Reames [1993] reported very high ${ }^{3} \mathrm{He} /{ }^{4} \mathrm{He}$ ratios ranging from 0.1 to 20 in those events. These large ratios were explained by a model in which the ${ }^{3} \mathrm{He}$ ions were preferentially accelerated by a wave-resonance mechanism [Fisk, 1978; Temerin and Roth, 1992] during solar flares. Data from the WIND spacecraft on August 27, 1996 showed that : (1) the solar wind ion density decreased from $15 / \mathrm{cm}^{3}$ at 8 UT to $10 / \mathrm{cm}^{3}$ at $10 \mathrm{UT}$; (2) the solar wind speed fluctuated between $430 \mathrm{~km} / \mathrm{s}$ and $455 \mathrm{~km} / \mathrm{s}$ during the two-hour period; (3) a small 20-minute SEP event was detected at $4 \mathrm{UT}$ with a peak helium flux of 22 particles $/\left(\mathrm{cm}^{2}\right.$-sr-s-MeV/n) at $0.32-0.64 \mathrm{MeV}$ energies. While in the above small SEP event the helium energy is comparable to that in Figure 1, this SEP event occurred 4.5 hours earler than the polar event, and both the time period and peak flux in the SEP event were almost one order of magnitude less than that in the polar event. In other words, no simultaneous comparable flux was observed by the WIND spacecraft, and this seems to rule out the solar flare as a direct and transient source.

To gain an insight into what factors are important to confine $\mathrm{MeV}$ ion fluxes in the polar region, in Figure 4 we plot the magnetic field direction angles and

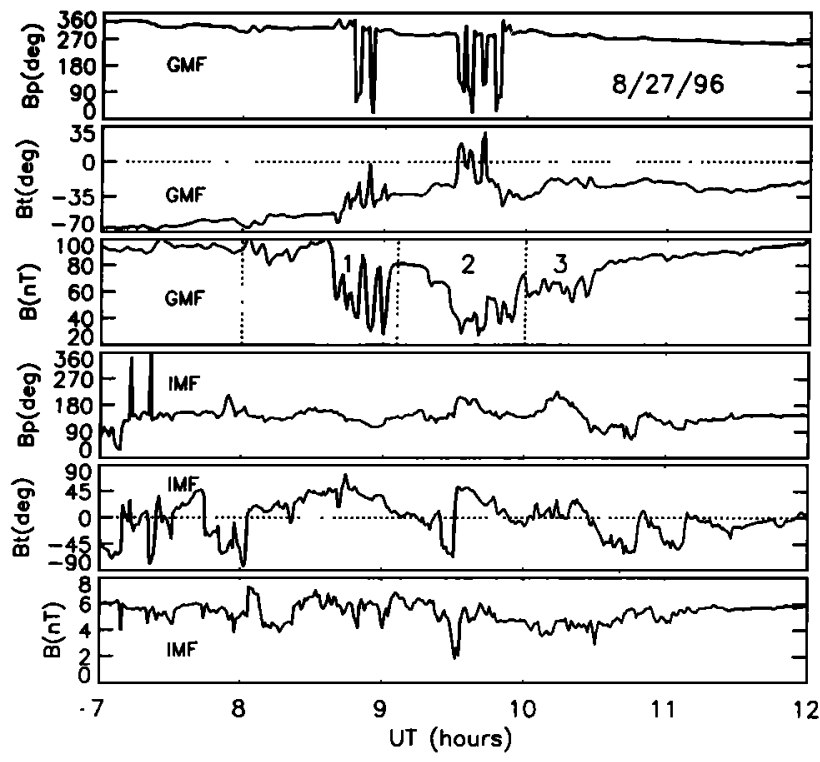

Figure 4. Magnetic field directions and magnitude, in the GSM polar coordinates, for both the GMF (top three panels), measured by POLAR, and the IMF (bottom three panels), measured by WIND, where $B p$ is the longitude; $B t$, the latitude; and $B$, the magnitude. The numbers of 1,2 , and 3 within the vertical dashed lines mark the three different regions in the event.

magnitude during the period of interest in the Geocentric Solar Magnetospheric (GSM) polar coordinates. Figure 4 shows that within the two-hour event period the geomagnetic field (GMF) experienced strong distortions, which have no obvious temporal association in the IMF. The WIND spacecraft was in a position about $90 R_{e}$ from the Earth during the event period, and one would expect a 22 minutes delay with the observed solar wind speed for any causal variation. To confine energetic ions, two local maxima of the geomagnetic field intensities are required or at least a localized minimum field intensity. Indeed, Figure 4 shows three local GMF $B$-minima, and this may substantiate the idea of the observed temporary confinement.

The MICS data indicate that POLAR was in a region dominated by magnetosheath plasma. The orbit showed that in this time interval, POLAR was just above the nominal position of the cusp. The IMF, as shown in Figure 4, was northward for most of the interval, which would move the cusp region northward and possibly open up the "throat" of the field topology making the magnetosheath plasma accessable to POLAR. A detailed inspection on GMF $B$ panel in Figure 4 and $\mathrm{He}^{++}$panel in Figure 3 reveals that the three local GMF minima are anticorrelated with the local $\mathrm{He}^{++}$ peak fluxes. However, this is not the case for the higher energy helium measured by the HIT.

\section{Summary and Conclusions}

The HIT sensor onboard the POLAR spacecraft observed a two-hour energetic heavy ion event $(\sim 1 \mathrm{MeV})$ at 8:25-10:25 UT on August 27, 1996 near apogee ( $~ 9$ $R_{e}$ and an invariant latitude of $\sim 80^{\circ}$ ). Our principal 
conclusions are the following: (1) Unique observation of very energetic helium in vicinity of day-side polar cusp, which showed three different helium peaks (or regions). (2) The helium pitch angle distribution in these three regions are different from each other, and all of them are different from an isotropic distribution. (3) The event had a peak spin averaged flux of nearly 150 particles/( $\mathrm{cm}^{2}$-sr-s-MeV $)$. No comparable flux was observed by the WIND spacecraft. (4) The appearance of the lower energy $\mathrm{He}^{++}$and $\mathrm{O}^{>+2}$ indicates a likely solar source for this event. (5) From region 1 to 2 to 3 in Figure 3 , the helium energy spectra progressively softened. (6) A distorted magnetic field with three local minima corresponding to the three helium peak fluxes was observed by POLAR during this two-hour period. One possible explanation of the event is that these ions were energized from lower energy helium by a local acceleration mechanism that preferred smaller rigidity ions. A new temporarily confined and solar originated ion population (up to at least $2 \mathrm{MeV}$ ) in the polar cusp, which exists only during GMF distortion periods, has been observed.

Acknowledgments. We want to acknowledge the contribution of B. Laubscher, R. Hedges, R. Vigil, and G. Lujan on the CAMMICE HIT sensor system at the Los Alamos National Laboratory; R. Koga, P. Lew, N. Katz, and B. Crain on the HIT data processing unit at the Aerospace Corporation; and the admistrative support and interest provided by D. Cobb at the Los Alamos National Laboratory. We are grateful to R. Lepping for providing us the WIND IMF data and $C$. T. Russell for the POLAR GMF data. This research was supported by NASA grant NAG5-2578.

\section{References}

Axford, W. I., On the origin of radiation belt and auroral primary ions, in Particles and Fields in the Magnetosphere, edited by B. M. McCormac, p. 46. D. Reidel, Dordrecht, Netherlands, 1970.

Chen, J. et al., Energetic helium particles trapped in the magnetosphere, Geophys. Res. Lett, 21, 1583, 1994.

Chen, J. et al., Geomagnetically trapped energetic helium nuclei, in Workshop on the Earth's Trapped Particle Environment (Taos), edited by G. D. Reeves, Amer. Instit. of Phys. Conf. Proc. No. 383 (Woodbury, NY), AIP Press, p. 161-167, 1996a.

Chen, J. et al., Energetic helium isotopes trapped in the magnetosphere, J. Geophys. Res., 101, 24787, 1996b.

Cornwall, J. M., Radial diffusion of ionized helium and protons: A probe for magnetospheric dynamics, J. Geophys. Res., , 77, 1756, 1972.
Fisk, L. A., ${ }^{3}$ He-rich flares: A possible explanation, Astrophys. J., , 224, 1048, 1978.

Geiss, J. et al., Apollo 11 and 12 solar wind composition experiments: Fluxes of $\mathrm{He}$ and $\mathrm{Ne}$ isotopes, J. Geophys. Res., 75, 5972, 1970.

Geiss, J., and H. Reeves, Cosmic and solar system abundances of deuterium and helium-3, Astronomy and Astrophys., 18, 126, 1972.

Krimigis, S. M., and J. A. Van Allen, Geomagnetically trapped alpha particles, J. Geophys. Res., 72, 5779, 1967.

Fritz, T. A., and J. R. Cessna, ATS-6 NOAA low energy proton experiment, IEEE Trans. Aerospace and Electronic Systems, AES-11, No. 6, 1145, 1975.

Fritz, T. A., and S. M. Krimigis, Initial observations of geomagnetically trapped protons and alpha particles with OGO 4, J. Geophys. Res., 74, 5132, 1969.

Fritz, T. A. et al., The mass composition instruments (AFGL-701-11), in CRRES/SPACERAD Experiment Descriptions, eds. M. S. Gussenhoven, E. G. Mullen, and R. C. Sagalyn, Air Force Geophysics Laboratory Report AFGL-TR-85-0017 (Hanscom AFB, MA), p. 127, 1985.

Ilyin, V. D., I. V. Ilyin, and S. N. Kuznetsov, Stochastic instability of charged particles in a geomagnetic trap, Cosmic Res. 24, 75, 1986.

Reames, D. V., Non-thermal particles in the interplanetary medium, Adv. Space Res., 19 (No. 9), 331, 1993.

Selesnick, R. S., and R. A. Mewaldt, Atmospheric production of radiation belt light isotopes, J. Geophys. Res., 101, 19745, 1996.

Spjeldvik, W. N., and T. A. Fritz, Energetic heavy ions with nuclear charge $Z \geq 4$ in the equatorial radiation belts of the Earth: magnetic storms, J. Geophys. Res., 86, 2349, 1981a.

Spjeldvik, W. N., and T. A. Fritz, Observations of energetic helium ions in the Earth's radiation belts during a sequence of geomagnetic storms, J. Geophys. Res., 86, 2317, $1981 b$.

Temerin, M., and I. Roth, The production of ${ }^{3} \mathrm{He}$ and heavy ion enrichments in ${ }^{3} \mathrm{He}$-rich flares by electromagnetic hydrogen cyclotron waves, Astrophys. J., , 391, L105, 1992.

J. Chen, T. A. Fritz, R. B. Sheldon, H. E. Spence, and W. N. Spjeldvik, Center for Space Physics, Boston University, 725 Commonwealth Avenue, Boston, MA 02215. (e-mail: chen@buasta.bu.edu)

J. F. Fennell, The Aerospace Corporation, Los Angeles, CA 90009.

S. Livi, Max-Planck-Institut fuer Aeronomie, 37189 Katlenburg-Lindau, Germany

(Received October 10, 1996; revised January 27, 1997; accepted May 2, 1997.) 